\title{
Identifying the Source of a Sound
}

\author{
Torben Pastore had careers in rock n' roll, furniture design, and \\ construction before beginning a research career in psychoacoustics. Now \\ he investigates how humans perceive their acoustical surroundings. \\ By Rachel Berkowitz
}

A s a child, Torben Pastore of Arizona State University recalls hearing the soundscape of the woodlands and his parents' factory as music. After graduating Bowdoin College, Maine, with degrees in art and philosophy, he went on to be a touring psychedelic rock musician, a career that sparked his interest in acoustics. During those touring years, Pastore built a recording studio for himself and others in his apartment, using knowledge acquired from an acoustics textbook. But, he says, he concluded that he could never climb the "heights of intellect required for acoustics research." He changed his mind, however, when cancer curtailed his rock career in 2002, and then the 2008 economic recession destroyed his next career in construction.

During the recession, Pastore took whatever remodeling construction work he could get. At a client's party near

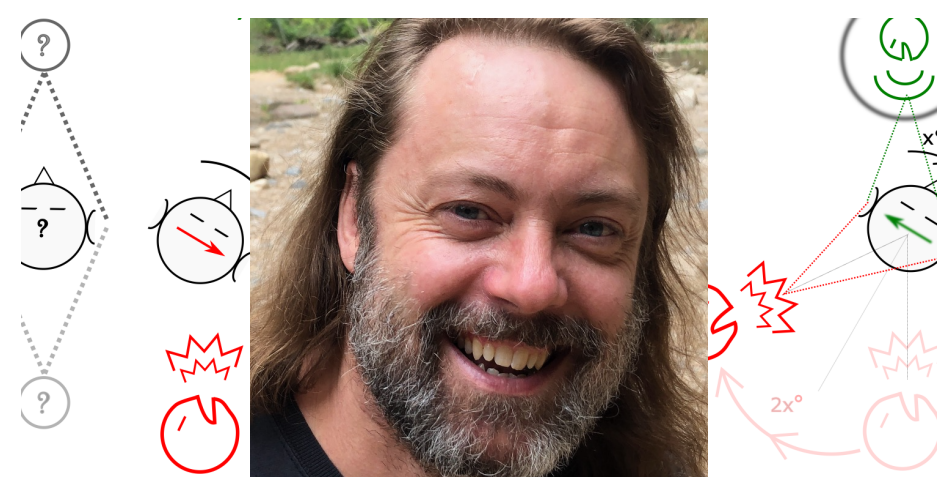

Credit: T. Pastore
Rensselaer Polytechnic Institute (RPI), New York, he discovered that the school had a program in architectural acoustics with a course in psychoacoustics, which looks at how auditory processing shapes human perception of the environment and how the built environment influences that perception. The psychoacoustics course description said auditory perception could be measured and the underlying mechanisms studied. Despite years of manipulating auditory perception through sound engineering, Pastore had no idea that such quantitative study was possible. In 2010, at age 40, Pastore was accepted as an RPI graduate student to study psychoacoustics and architectural acoustics. Recently, he completed a postdoc at Arizona State University, where he looked at how humans localize a moving sound source. Physics spoke to Pastore to learn about the unsolved problems in hearing science and why it's important to understand how humans localize sounds.

\section{All interviews are edited for brevity and clarity.}

\section{How do your careers in rock and research tie in together?}

Psychedelic musicians manipulate sounds to alter the listeners' perception of those sounds and to express things standard music cannot. Composing such music requires knowledge of signal processing, room acoustics, and sound engineering. I see the application of psychoacoustics to musical expression as a main component of psychedelic music.

\section{What problem in acoustics first captured your attention?}

Understanding how the brain makes sense of a complex 
auditory scene. It's an inverse problem: Imagine you're standing at the edge of a lake and are allowed to cut two shallow trenches into the mud, extending away from the lake and spaced a few feet apart. Using just the disturbance patterns in the water in these two trenches, you must determine what objects are out there floating on the lake and where exactly they sit. That's what the brain does all day.

\section{And what do you work on now?}

I'm working on a part of that problem: how exactly the brain pinpoints the location of a sound's source.

\section{How do you go about figuring that out?}

By playing sounds to a listener while manipulating the listener's position and movements. In the experiments I was involved in at Arizona State University, a blindfolded person was seated on a computer-controlled rotating chair and different sounds were played to them over a loudspeaker array. The person was then asked to report where they perceived the sound(s) to originate. I and my colleagues then tried to distinguish what brain processes led to the listener's auditory percept.

\section{How exactly did you do that?}

One way we did that was by using sound illusions. For example, in one set of experiments, I rotated a sound source around the rotating person. The source rotated at double the rate of the person. When the source produced no high-frequency sounds, clues were removed that can help indicate to a listener whether the source is in front of or behind them. Thus, the person received the same cues from that rotating source as they would from a stationary one. I then asked the person whether the sound was rotating or stationary. Manipulating the spectrum and rotation speed of the sound let me and my colleagues infer how the person processed signals when estimating a sound's location.

\section{What do your results show about how humans determine a sound's location?}

The overall outcome of this body of work is that sound source localization is a multisystem, multisensory process-it is not auditory alone.

\section{Why is it important to understand how people locate sounds?}

There are many groups interested in how the people locate sounds, from designers of virtual reality technologies to sound engineers to military aviation specialists to anyone who has lost function in one ear. The inputs to the two ears can only provide a listener with an estimate of a sound source's location relative to his or her head position. The person still needs to place that estimate into the context of the world around them. This conclusion may seem obvious, but how the brain accomplishes this feat remains opaque, although we think it involves the use of other senses and systems, such as sight, the somatosensory system, vestibular system, and even memory.

\section{What advice do you have for undergraduates interested in carrying out this type of research?}

It's not just the "how" that animates basic science, it's also the "why." I think art and philosophy cover that territory, and I rely on training from both every day. If I could start my science career over again, I would study science and engineering in a rigorous liberal arts environment, where the education extends the core subject of the major into the larger context of knowledge and culture.

Rachel Berkowitz is a Corresponding Editor for Physics based in Vancouver, Canada. 